\title{
De retour à Guennoc
}

Back to Guennoc

Sylvène Michel et Nicolas Naudinot

\section{(2) OpenEdition \\ Journals}

Édition électronique

URL : http://journals.openedition.org/rao/782

DOI : $10.4000 /$ rao. 782

ISBN : 978-2-7535-1609-0

ISSN : 1775-3732

Éditeur

Presses universitaires de Rennes

Édition imprimée

Date de publication : 31 décembre 2009

Pagination : 23-36

ISBN : 978-2-7535-1086-9

ISSN : 0767-709X

\section{Référence électronique}

Sylvène Michel et Nicolas Naudinot, « De retour à Guennoc », Revue archéologique de l'Ouest [En ligne], 26 | 2009, mis en ligne le 20 décembre 2011, consulté le 05 décembre 2020. URL : http:// journals.openedition.org/rao/782 ; DOI : https://doi.org/10.4000/rao.782 


\title{
De retour à Guennoc
}

\author{
Back to Guennoc
}

\author{
Sylvène Michel* et Nicolas Naudinot*
}

\begin{abstract}
Résumé : L'étude technologique de la collection lithique d'Enez Guennoc (Landéda, Finistère) a permis de compléter l'analyse et l'attribution chrono-culturelle proposée dans les années 1980. Loin d'être homogène, cette série témoigne d'un mélange entre Azilien et premier Mésolithique, auquel il faut rajouter le matériel néolithique lié aux cairns. La situation géographique de l'occupation, mise en relation avec la transgression flandrienne et ses conséquences sur la disponibilité des ressources naturelles, nous a permis d'esquisser l'organisation socio-économique de ces groupes de chasseurs-collecteurs en zone péri-côtière.
\end{abstract}

\begin{abstract}
Technological study of the Enez Guennoc assemblage (Landéda, Finistère, France) allows us to complete the chrono-cultural analysis proposed during the 80's. Culturally not homogeneous so far, the lithics show a mixture of Azilian and early Mesolithic groups, apart from Neolithic related to megalithic tombs. Geographical situation of settlement sea level changes and theirs consequences for resources procurement, allows us to draft a socio-economic model for such costal hunter-collectors societies.
\end{abstract}

Mots clés : Azilien, Finistère, prédation marine, premier Mésolithique, technologie lithique.

Key words: Azilian, early Mesolithic, Finistère, Lithic technology, Marine predation.

\section{INTRODUCTION}

Le site de Enez Guennoc (Landéda, Finistère) a fait l'objet de fouilles sous la direction de P.-R. Giot de 1960 à 1972, avec pour objectif premier la compréhension des cairns néolithiques (Giot, 1987). Au cours de ces opérations, des occupations antérieures ont été découvertes et attribuées à "l'Épipaléolithique azilien " (Giot et al., 1977; Giot, 1987; Monnier, 1980). L'association typologique des armatures a très vite convaincu $\mathrm{O}$. Kayser de l'expression d'un stade très ancien du Mésolithique, voire d'une étape de transition Pléistocène/Holocène (Kayser, 1984). Nous profitons du regain d'intérêt qui souffle sur l'îlot de Guennoc, comme en témoigne l'étude des occupations de l'Âge du fer (Daire, 2008), pour revenir sur les traces qu’y ont laissées les premières occupations humaines. Nous avons apporté un regard technologique sur cette série lithique afin d'en affiner le dia- gnostic culturel et de mieux connaître les systèmes socio-économiques de ces derniers groupes de chasseurs-collecteurs. Par ailleurs, l'occupation néolithique de l'île ne semble pas s'étendre en dehors du contexte sépulcral, cette composante n'apparaissant pas véritablement dans la collection dont il est question ici.

\section{UNE INSTALLATION SUR UN PROMONTOIRE DOMINANT L'ESTUAIRE DE L'ABER BENOÎT}

L'actuelle île Guennoc, promontoire rocheux d'environ $400 \mathrm{~m}$ de long pour $160 \mathrm{~m}$ de large, est constituée de migmatite de Plouguerneau et de granite migmatitique avec des variations de faciès possibles entre gneiss et granite porphyroïde (Giot, 1987). Le sol, peu développé et probablement érodé, se résume souvent à une simple humification de

*UMR 6566 CreAAH Laboratoire lithique Université de Rennes 1 - Bâtiments 24, Campus de Beaulieu 35042 Rennes Cedex. 
quelques centimètres d'épaisseur. Ce phénomène complique d'autant notre vision des niveaux les plus anciens mélangés aux occupations postérieures.

Actuellement au débouché de l'Aber Benoît dans la Manche, l'île Guennoc est un dôme de 8 à 16 m NGF entouré de falaises de 2 à $3 \mathrm{~m}$ (fig. 1). La transgression flandrienne a modifié le trait de côte durant la Préhistoire. Ainsi, selon D. Menier (2004), le niveau de la mer durant le Pléniglaciaire se trouvait en Bretagne nord à $-120 \mathrm{~m}$ par rapport au niveau actuel de la Manche (18000 BP). Il sélève à $-65 \mathrm{~m}$ durant le Dryas récent et à $-25 \mathrm{~m}$ il y a 8000 ans (ibid.). D'après les courbes bathymétriques publiées par le Service hydrographique et océanographique de la Marine (carte $n^{\circ} 7094$ ), on peut estimer que Guennoc était rattachée au continent au premier Mésolithique (Préboréal-Boréal), la mer étant alors distante d'environ $4 \mathrm{~km}$. L'occupation se trouvait néanmoins dans une zone à forte influence marine, de par sa position en bordure d'estuaire.

Les fouilles ont permis d'appréhender l'ensemble des structures apparentes de Guennoc, à commencer par les cairns néolithiques. L'industrie lithique associée à ces mégalithes n'a pas été réétudiée dans cet article. Elle n'est pas abondante, se résumant à quelques éclats ou fragments de lames en silex et un petit nombre d'outils comme des grattoirs sur éclats, quelques burins et perçoirs, des lames de haches polies ainsi qu'une flèche à pédoncule et ailerons (Giot, 1987). Les témoignages de l'Âge du Fer sont également très présents sur l'île (Daire, 2008). Cet attrait pour Enez Guennoc a perduré aux cours des siècles avec des indices d'occupations médiévales, modernes et contemporaines. Le soin apporté aux fouilles de l'époque, avec notamment un tamisage systématique des sédiments, nous a permis de bénéficier d'une série exhaustive indispensable au bon déroulement de l'étude lithique.

Cinq zones de fouille ont livré une industrie antérieure au Néolithique, aux alentours du cairn II et de l'enclos ou sous ces structures (fig. 2). Nous n'avons cependant pas réussi à attribuer clairement les différentes pièces découvertes à l'une ou l'autre de ces cinq zones, d'où l'impossibilité d'effectuer une analyse spatiale. Le "vieux sol» a tout d'abord été considéré comme un gisement "épipaléolithique azilien " au matériel homogène (Giot et al., 1977; Monnier, 1980; Giot, 1987). Dans le cadre des travaux sur le Mésolithique finistérien, la collection lithique a ensuite été attribuée au Mésolithique ancien à partir des planches de dessin des ouvrages précités (Kayser, 1984; Gouletquer et al., inédit).

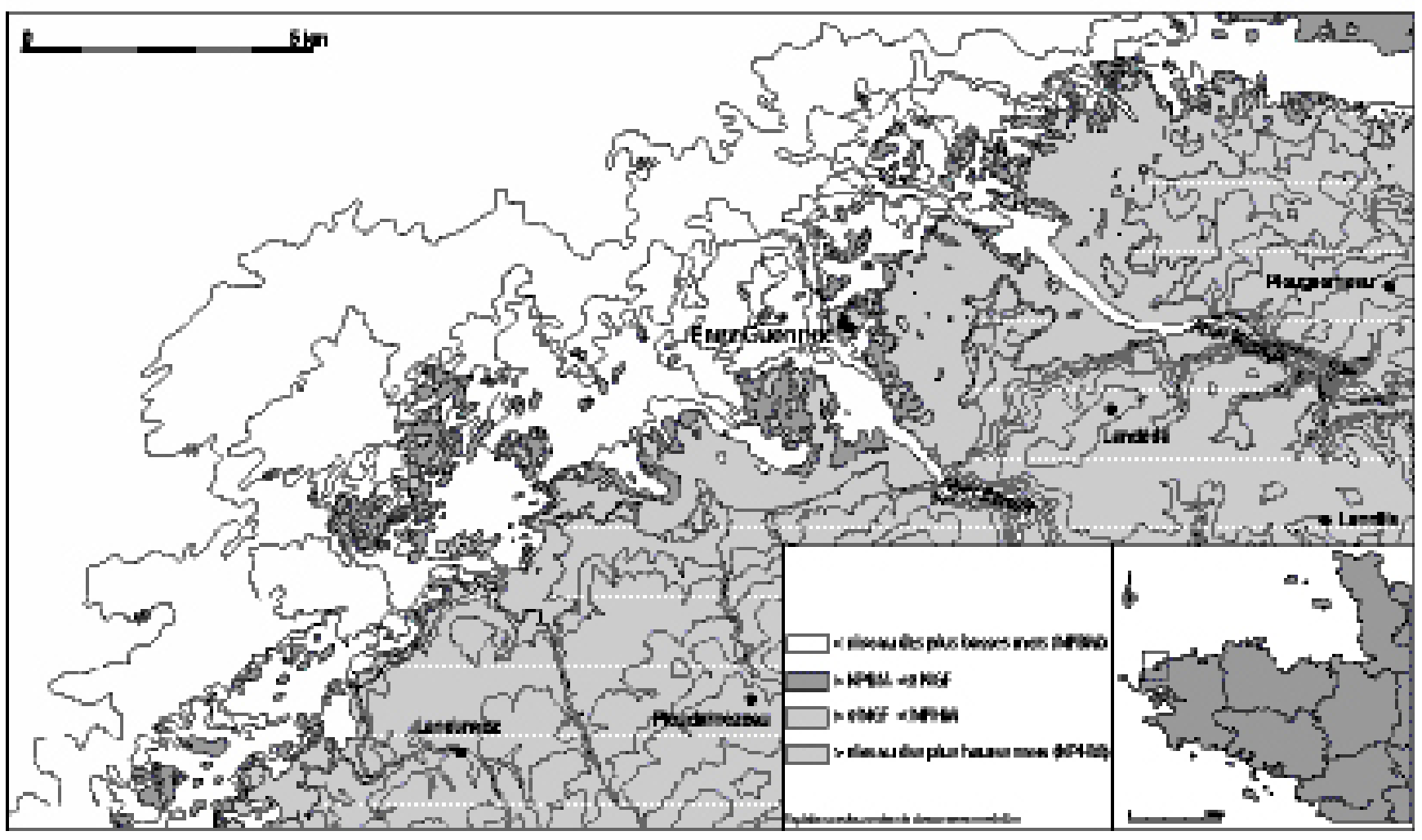

Figure 1 : Localisation, topographie et bathymétrie des environs du site de Guennoc (DAO L. Quesnel et M.-Y. Daire d'après les fonds cartographiques du SHOM et de l'IGN).

Figure 1: Situation, topography and bathymetry of the Guennoc area. 
Figure 2 : Guennoc : situation des zones fouillées en partie centrale de l'île.

Figure 2: Excavated areas in the island center.

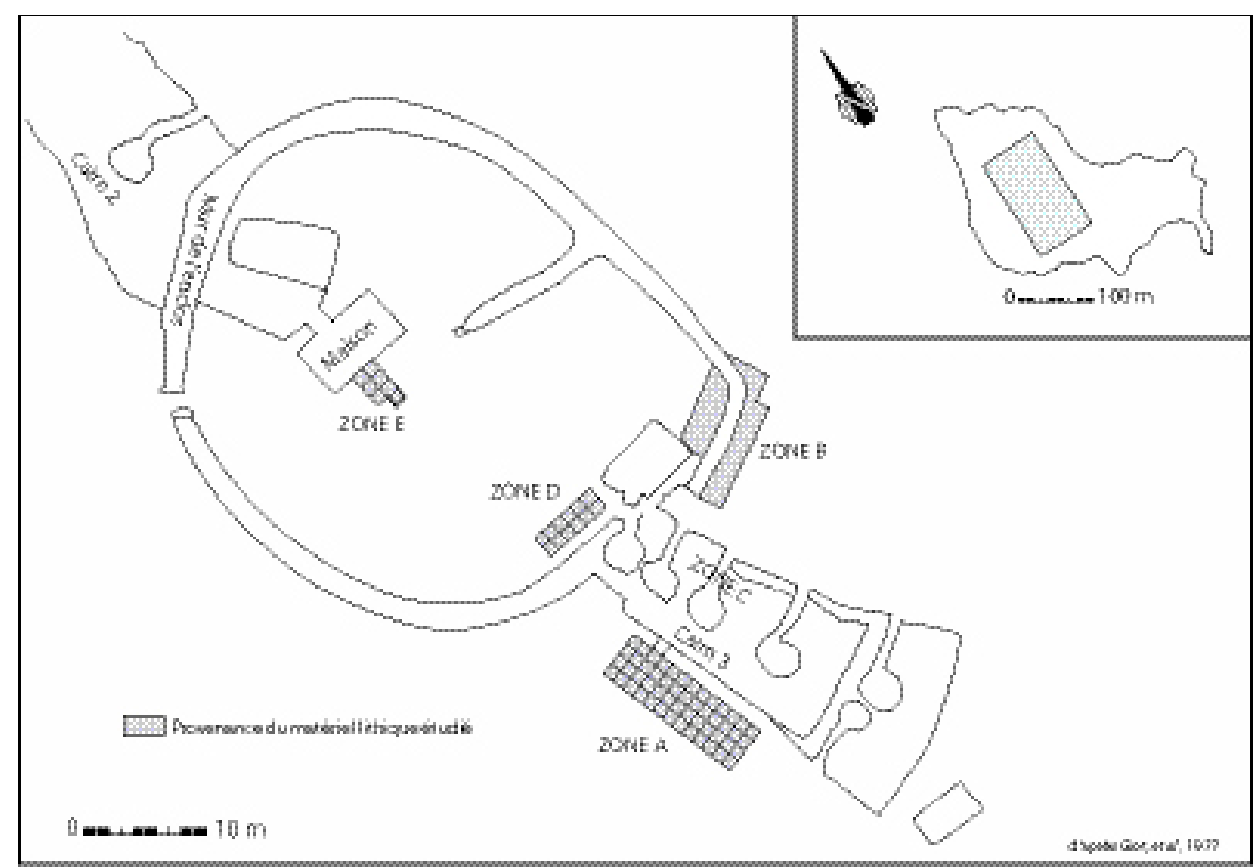

L'observation de la collection montre cependant l'existence d'un mélange associant ces deux attributions. Les perturbations post-dépositionnelles, avec une forte intervention des animaux fouisseurs ainsi que des témoignages d'agriculture récente, n'ont pas permis de distinguer différentes couches d'occupations à la fouille, ce qui rend l'étude des industries lithiques encore plus ardue.

\section{LES PREMIÈRES OCCUPATIONS HUMAINES DE GUENNOC : ANALYSE DU MATÉRIEL LITHIQUE}

Croire que l'ensemble du matériel lithique va être significatif dans le cadre d'une attribution chronologique est utopique. Ainsi proposons-nous, en nous basant sur certaines pièces favorables à la diagnose chrono-culturelle, d'avancer pas à pas pour débroussailler un épineux mélange lithique.

\section{Enquête sur l'outillage et les armatures}

Grâce au tamisage effectué, nous bénéficions d'un corpus d'armatures reflétant assez bien les carquois des différents occupants de Guennoc (fig. 4 et 5). Cependant, la forte fragmentation des pointes de projectile (à $96,2 \%$ ), encore plus marquée que pour le reste du débitage $(80,6 \%)$, certainement en raison de la finesse des lamelles, rend l'identification des types parfois complexe. Leurs supports sont systématiquement dépourvus de cortex et auraient été extraits lors des séquences lamellaires de plein débitage.
La moitié des armatures sont des lamelles à bord abattu. La délinéation du bord est essentiellement rectiligne, même si quelques rares exemplaires convexes pourraient être rattachés à des parties basales ou mésiales de monopointes à dos courbe. Ces pièces correspondent peut-être en partie à des pointes à dos, mais leur fragmentation nous interdit de nous prononcer de façon catégorique. Notons également qu'une de ces armatures, au bord denticulé, semble abandonnée en cours de fabrication. Une autre porte une fracture en cône sur la face supérieure du bord abattu, témoignant d'un accident lors de la retouche croisée du support. La latéralisation du dos est essentiellement senestre (à 69,6 \%) lorsque la pièce est orientée partie proximale en bas. La largeur moyenne de ces lamelles une fois retouchées est de $8 \mathrm{~mm}$ pour une épaisseur de 3,2 $\mathrm{mm}$. Ces différents fragments ne présentant pas la totalité des critères morphologiques et techniques permettant une diagnose, ils ne peuvent donc pas être utilisés comme marqueurs chrono-culturels.

Les sept armatures classées comme monopointes à dos courbe sont attribuables à l'Azilien récent, daté de l'Allerød (12000-11 000 cal. BC). Seules les pièces présentant un apex ont été rattachées à cette classe; cependant, trois fragments de pièces à dos portant une fine retouche sur le bord opposé à une extrémité pourraient être identifiés comme des bases de pointes de Grundy. Ils peuvent également être rapprochés d'un point de vue technique des penknife points anglaises contemporaines. La proximité des îles britanniques à ce stade de la transgression marine rend cette comparaison séduisante, même si des aménagements très opportunistes liés à l'emmanchement sont souvent observés sur des mono- 


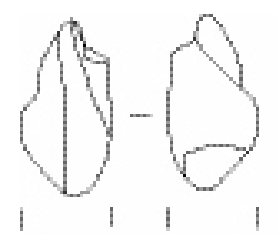

1

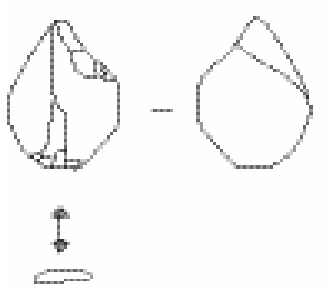

6

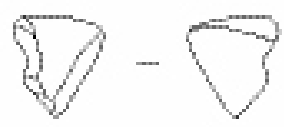

10

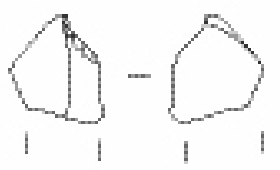

2

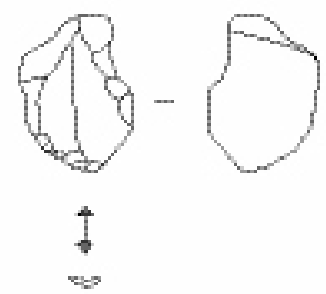

7

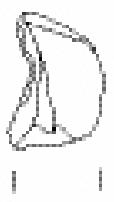

11

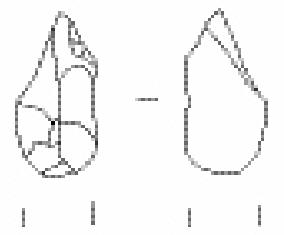

3

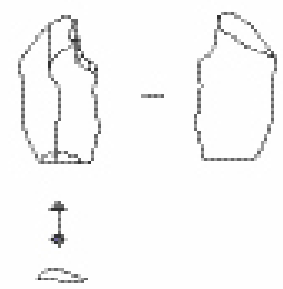

8

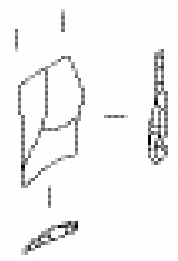

12
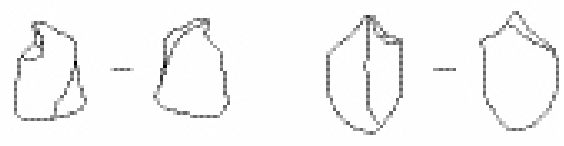

4

5

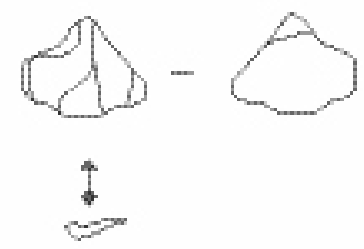

9

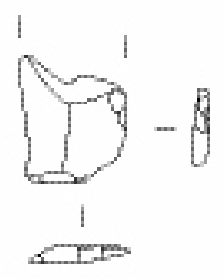

13

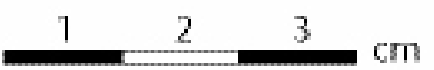

Figure 3 : Microburins : 1-11 (dont 10 et 11 avec cassures au dessus de la coche); 12 : triangle scalène fragmenté; 13 : fragment d'armature géométrique.

Figure 3: Microburins: 1-11 (10 \& 11 with fracture at top of the notch); 12: broken scalene triangle; 13: geometric microlith fragment.

pointes à dos courbe, y compris plus au sud comme sur le site des Prises (Brizambourg, Charente-Maritime : Naudinot in Blanchet et al, 2007).

Une pointe à dos rectiligne et apex aménagé par troncature très oblique a également été identifiée. Elle pourrait correspondre aux canons des pointes de la Gravette mais pourrait également être rapprochée des pointes des Blanchères (Rozoy, 1978), découvertes en contexte de transition Dryas récent/ Préboréal. Cependant ses dimensions s'en écartent avec une largeur de $10 \mathrm{~mm}$ pour une épaisseur de $5 \mathrm{~mm}$ (alors que les moyennes sont respectivement de 6 et $2 \mathrm{~mm}$ sur les pointes des Blanchères du Camp d'Auvours [Allard, 1982; Naudinot, 2008) et que l'orientation de la pointe en extrémité distale est assez anecdotique parmi ce type d'armatures.

Si le procédé de la troncature oblique est constamment utilisé au cours du premier Mésolithique, de récents travaux montrent qu'on assiste à sa généralisation dès la transition avec le Dryas récent. L'observation de monopointes à dos courbe abandonnées en cours de fabrication plaiderait en faveur d'une utilisation dès l'Allerød (Valentin et Hantaï, 2005). Dans le corpus étudié, les pointes à troncature oblique sont bien représentées $(27,8 \%)$, avec une obliquité plus ou moins marquée. Elles sont essentiellement latéralisées à gauche $(61,1 \%)$, la troncature s'effectuant majoritairement en partie proximale $(69,2 \%)$; ces deux critères sont d'ailleurs récurrents sur ces armatures dans le premier Mésolithique régional. Ce sont de petites lamelles, peu larges et peu épaisses (respectivement 8,6 et 1,9 $\mathrm{mm}$ en moyenne), qui ont été sélectionnées comme support. On note une différence très marquée avec les lamelles à bord abattu en ce qui concerne l'épaisseur. 


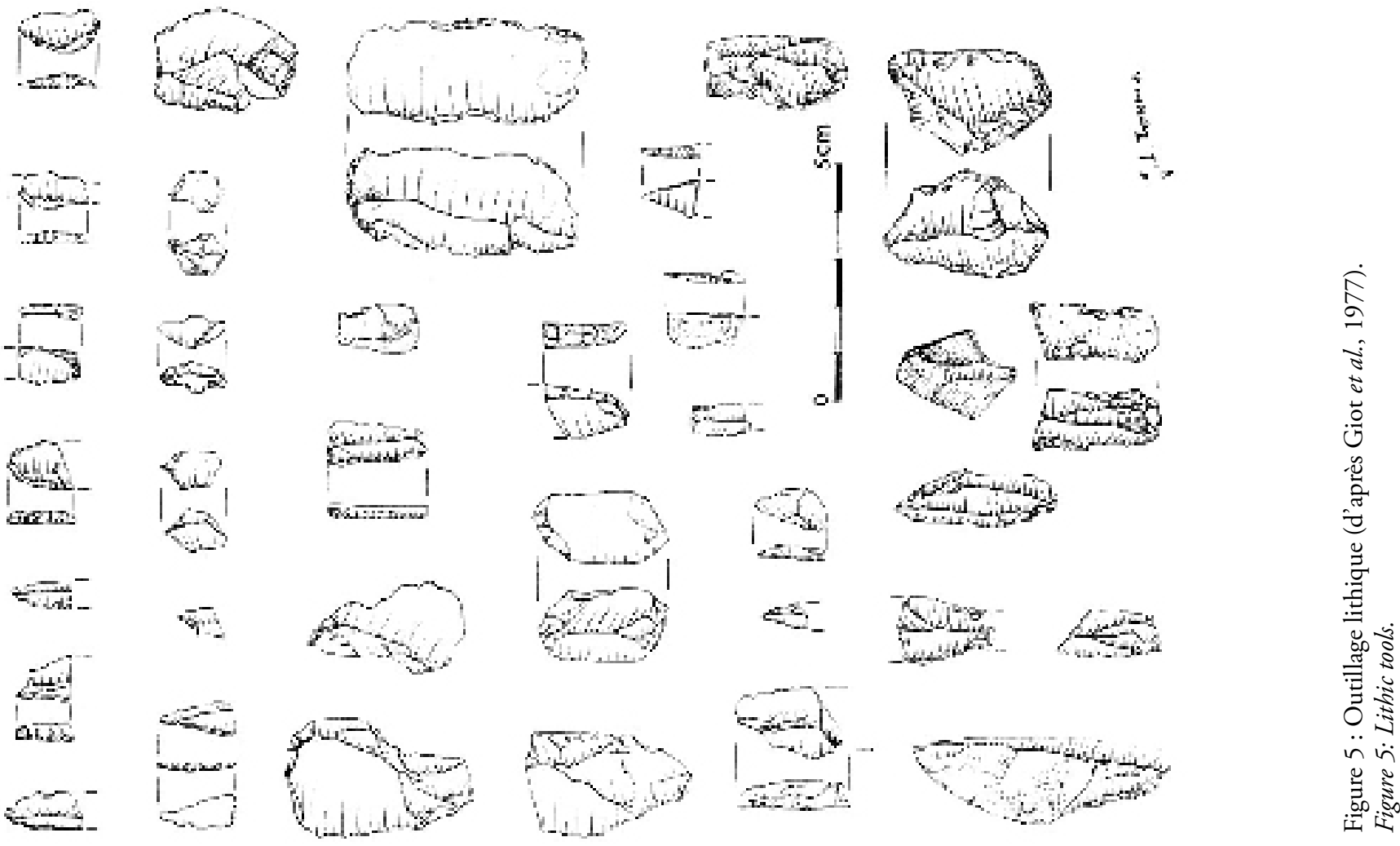

trit

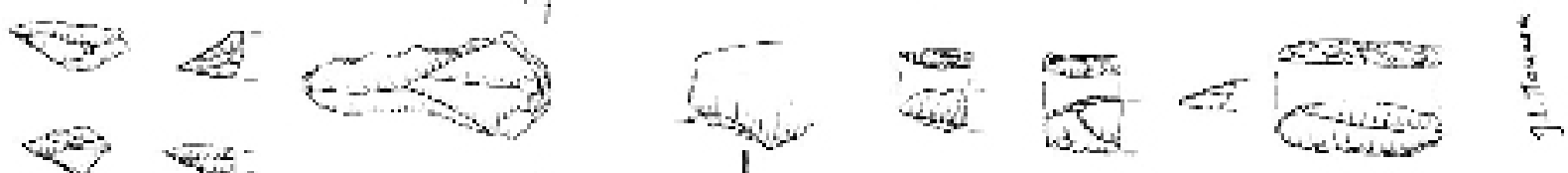

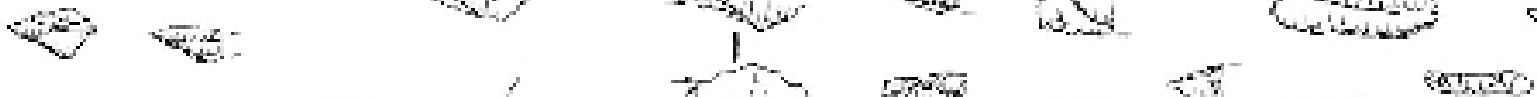

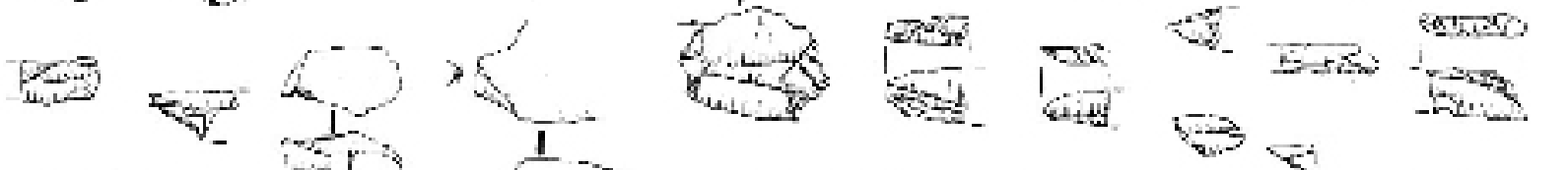
过

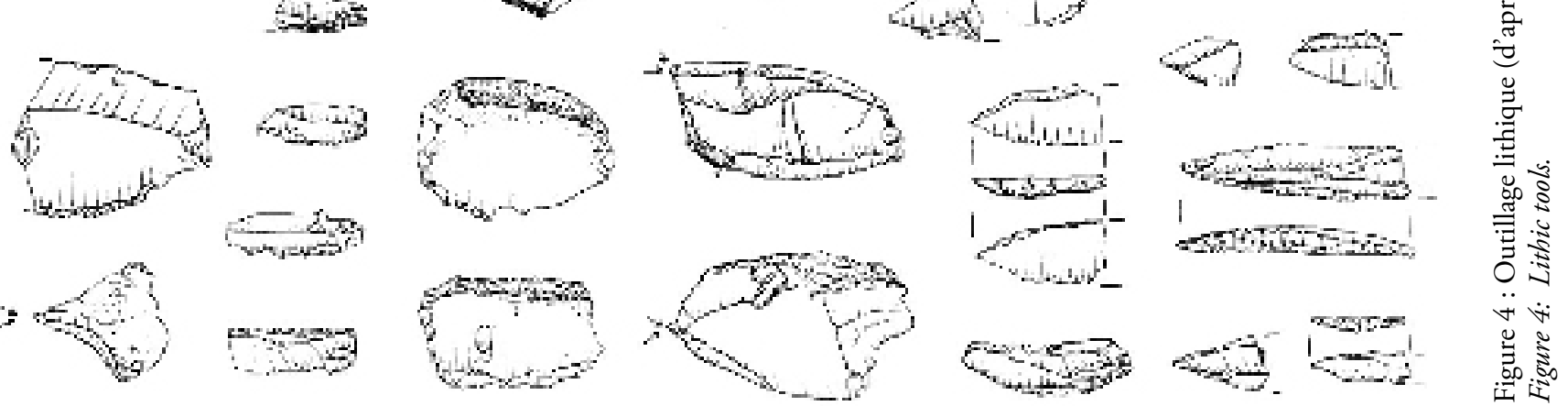


Les microlithes géométriques sont très rares à Guennoc; on n'y compte en effet qu'un seul triangle scalène. À sa petite troncature concave s'ajoute une grande troncature brisée, sinueuse et gibbeuse, témoignant certainement d'un abandon en cours de fabrication. Terminé, ce triangle scalène de petites dimensions $(1.5 \mathrm{~mm}$, e. $1,5 \mathrm{~mm})$ aurait été latéralisé à droite. Aucune trace de piquant trièdre n'est visible, ce qui n'exclut pas le recours au procédé du microburin dont l'utilisation est attestée sur le site (fig. 3). Alors qu'on note un certain équilibre entre microburins proximaux et distaux, leur latéralisation à droite est quasi systématique (91,7\%).

Dix pointes de projectiles ont été classées comme armatures indéfinies. Il est intéressant de noter que trois de ces pièces pourraient venir gonfler le corpus des microlithes géométriques. Le mélange des différents niveaux d'occupation pourrait être confirmé ici par la présence d'une éventuelle flèche tranchante néolithique.

L'outillage commun (fig. 4 et 5) est faiblement représenté dans la série et est essentiellement réalisé sur des supports issus des premiers stades de débitage ou de l'entretien des volumes, comme en témoigne la présence de cortex sur un tiers de ces outils. La classe la plus importante est constituée de supports à retouches diverses et de troncatures (tabl. 1). Ces dernières, sur supports lamino-lamellaires, sont aussi bien obliques (à droite) que transverses; leur délinéation est majoritairement rectiligne et l'aménagement se fait préférentiellement en partie distale du support. Ces pièces sont accompagnées d'autres classes minoritaires : grattoirs sur éclats (dont un très petit de 13,7 x 14,9 x 7,3 mm), encoches et burins sur cassure ou pan naturel. Une lame à néocrête semi-corticale portant une troncature oblique convexe associée à un amincissement de la basse pourrait être un couteau à dos de type indéterminé. L'outillage commun ne permet donc pas d'affiner le diagnostic culturel. On peut tout de même souligner l'absence de perçoirs et le fort taux de troncatures, ce qui écarterait l'hypothèse d'une forte composante néolithique.

\begin{tabular}{|l|c|}
\hline & Effectif \\
\hline Burin & 3 \\
\hline Grattoir & 4 \\
\hline Coche & 3 \\
\hline Retouche divers & 15 \\
\hline Troncature & 11 \\
\hline Aposteriori & 15 \\
\hline
\end{tabular}

Tableau 1 : Guennoc : composition générale de l'outillage. Table 1: General count of preneolithic tools.

\section{Analyse technologique}

La totalité des pièces issues des cinq zones de fouilles a été étudiée suivant des critères morphométriques et morpho-technologiques. Nous proposons ici de décrire la chaîne opératoire depuis l'approvisionnement en matière première jusqu'à l'utilisation des outils façonnés. Nous sommes cependant bien conscients qu'étant donné l'hétérogénéité de la série, différentes chaînes opératoires doivent se fondre dans cet ensemble.

L’ensemble lithique est composé de 1817 pièces (tabl. 2), dont $25,7 \%$ présentent une altération thermique. Mais des activités liées au feu au cours des différentes phases d'occupation ultérieures (foyer médiéval, four à goémon...), ainsi qu'une "combustion lente [de l'île] pendant des mois" en 1953 (Giot, 1987) nous invite à prendre du recul sur ce chiffre.

La série est essentiellement constituée de silex (à 98,5 \%). Au sein de cet ensemble, 12,9\% des pièces présentent des vestiges corticaux d'aspect roulé. Ces surfaces sont parsemées d'impacts pouvant résulter d'un transport maritime et par conséquent traduit un approvisionnement en position secondaire sur le littoral voisin. Une faible proportion d'éléments $(0,5 \%)$, au cortex natif, pourrait avoir fait l'objet d'une collecte en position primaire; l'origine en reste indéterminée. Les autres pièces en silex sont dépourvues de

\begin{tabular}{|l|c|c|}
\hline & Effectif & $\%$ \\
\hline Éclat a-cortical & 174 & 9.58 \\
\hline Éclat cortical & 36 & 1.98 \\
\hline Éclat semi-cortical & 66 & 3.63 \\
\hline Éclat d'avivage, éclat à néo-crête & 10 & 0.55 \\
\hline Lame à néo-crête & 10 & 0.55 \\
\hline Lamelle à néo-crête & 9 & 0.50 \\
\hline Réaménagment du PDF & 5 & 0.28 \\
\hline Lame a-corticale & 205 & 11.28 \\
\hline Lame corticale & 5 & 0.28 \\
\hline Lame semi-corticale & 49 & 2.70 \\
\hline Lamelle a-corticale & 448 & 24.66 \\
\hline Lamelle corticale & 10 & 0.55 \\
\hline Lamelle semi-corticale & 27 & 1.49 \\
\hline Nucleus & 24 & 1.32 \\
\hline Casson & 194 & 10.68 \\
\hline Chute de burin & 3 & 0.17 \\
\hline Esquille & 542 & 29.83 \\
\hline Total & 1817 & 100 \\
\hline
\end{tabular}

Tableau 2 : Composition générale de la série. Table 2: Total count of Guennoc. 
cortex, mais leurs teintes évoquent celles des galets de silex côtier de la région, originaires du Crétacé de la Manche. Les autres matières présentes à Guennoc sont anecdotiques (Quartz hyalin et filonien, quartzite et grès lustré), et le caractère intentionnel de leur fracture n'est pas toujours vérifiable. Il faut donc souligner ici la préférence accordée au silex aux dépens des autres roches taillables disponibles dans la région. Pour indication, la totalité de l'outillage commun et des armatures est en silex.

Des activités de mise en forme des blocs se sont déroulées sur place au cours d'une ou de plusieurs occupation(s) préhistorique(s), si l'on s'en réfere au taux de pièces (semi-) corticales $(13,4 \%)$. Le décalottage des galets par percussion bipolaire sur enclume ne semble pas appartenir au bagage technique des tailleurs; on compte tout de même deux supports présentant des caractéristiques de ce débitage. Les crêtes d'initialisation n'interviennent pas dans la mise en forme, mais les tailleurs semblent plutôt avoir profité des convexités naturelles des galets. En effet, on ne compte que deux crêtes portant les vestiges de plages corticales, ces dernières pouvant résulter d'une extension du débitage sur les flancs, et ce même dans les dernières séquences d'exploitation.

La série est à composante essentiellement lamino-lamellaire (72,9\%), dont deux tiers de lamelles (fig. 6). La fragmentation est particulièrement importante puisqu'elle atteint $78,3 \%$. La largeur des produits est surtout concentrée entre 6 et $11 \mathrm{~mm}$, ce qui, pour les plus étroits, correspond aux derniers négatifs visibles sur les nucléus (fig. 7). Contrairement aux lamelles, le diagramme de production des lames montre une exploitation plus dispersée; nous préférons cependant rester prudents quant à l'interprétation de ce résultat étant donné les importants mélanges de différents techno-complexes. Quelques remontages ont permis d'affiner nos observations. Un de ces ensembles montre une réduction des volumes où les séquences lamellaires sont intercalées dans les séquences laminaires, mais ce seul exemple ne nous autorise pas à généraliser cette modalité qui n'en exclut pas d'autres. La bipolarité est plus marquée sur les lames que sur les lamelles (fig. 8) ce qui, dans une chaîne opératoire unique, indiquerait un rythme d'alternance plus marqué dans les premières phases de débitage. Les lames à deux et trois pans sont également réparties (respectivement $53 \%$ et $47 \%$ ), alors que les lamelles possèdent majoritairement une seule nervure (à $65 \%$ ).

En ce qui concerne les techniques de percussion, l'étude des talons montre que leurs types diffèrent en fonction des supports. Ainsi, les éclats ont souvent été extraits à partir de plans de frappe corticaux alors que les lames et lamelles présentent des talons plutôt lisses minces, filiformes et punctiformes (fig. 9). L'usage d'un percuteur de pierre dure est attesté pour les éclats, alors que les produits lamino-la- mellaires portent souvent une lèvre bien marquée (fig. 10), plaidant plutôt en faveur d'un plein débitage mené au percuteur tendre organique. Cependant, la présence de quelques esquillements du bulbe pourrait être révélatrice d'un recours à un percuteur tendre minéral. Cette dichotomie des stigmates pourrait également être perçue comme un changement d'orientation du geste de percussion (de rentrant à tangentiel) au cours de l'exploitation au percuteur de pierre tendre. Mais n'oublions pas que ces observations peuvent être le reflet de différentes composantes culturelles au sein de l'assemblage.

Certaines des pièces étudiées traduisent une volonté d'entretien du nucléus au cours de ses différentes phases d'exploitation; c'est le cas des 17 néo-crêtes, essentiellement partielles à un pan. Le ravivage des plans de frappe n'est quant à lui que très peu attesté à Guennoc. La rareté des nucléus à éclats ainsi que la proportion d'éclats portant les vestiges de plages corticales témoigneraient de leur implication dans les phases techniques, comme la mise en forme ou l'entretien des convexités, plutôt que d'une production indépendante; le cas des éclats lamellaires est quelque peu différent ( $c f$. infra).

Parmi les 24 nucléus récoltés, deux ensembles s'individualisent par leur objectif de débitage (fig. 11 à 13). Les trois nucléus à éclats présentent de multiples changements d'axe de débitage et une exploitation de la totalité des faces du volume. Des réfléchissements survenant en phase finale de débitage semblent à l'origine de l'abandon de certains nucléus. Des inclusions mal silicifiées sont également à l'origine de l'arrêt de l'exploitation. Les nucléus à petites lamelles étroites/éclats lamellaires forment quant à eux la majorité du corpus. La production de lamelles étroites semble indissociable de celle de ces petits éclats, ces deux types de produit ne formant qu'un seul et même objectif. Un seul nucléus privilégie le premier type de produit (fig. $11, \mathrm{n}^{\circ} 1$ ) : son unique table unipolaire s'implante dans la longueur d'un galet côtier, le débitage à partir d'un plan de frappe lisse à corniche finement abrasée est semi-tournant et déborde sur les flancs; le dos est partiellement laissé cortical et un problème de convexité, accentué par un fort réfléchissement, est certainement à l'origine de l'abandon du bloc. Les nucléus associant production de lamelles et de petits éclats suivent différents modèles. Leur interprétation en terme de modalités de débitage pose problème : a-t-on affaire à différentes modalités ou à l'expression de plusieurs stades d'exploitation d'une même modalité? Les différentes gestions du volume des blocs et manières de mener le débitage peuvent se résumer ainsi :

- exploitation partielle de galets marins qui conservent un dos cortical, sur une table gérée de manière frontale à semi-tournante à partir d'un unique plan de frappe, avec une éventuelle intervention d'un plan de frappe stricte- 

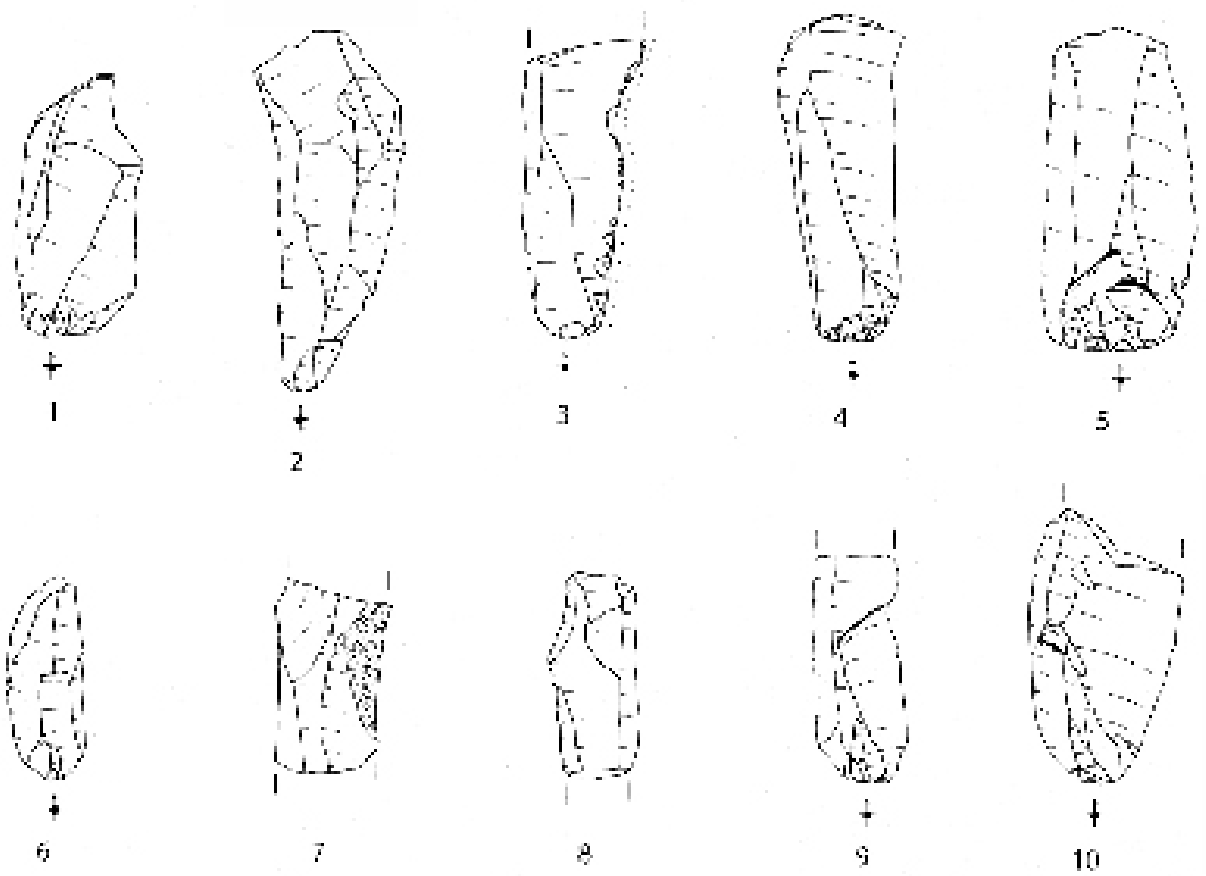

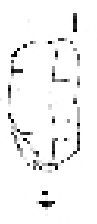

11

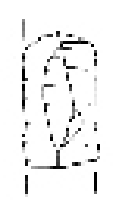

12

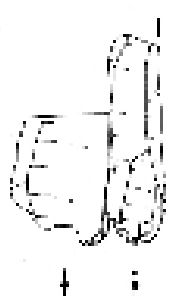

1.314

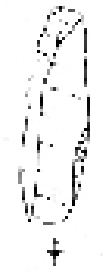

15

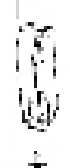

16

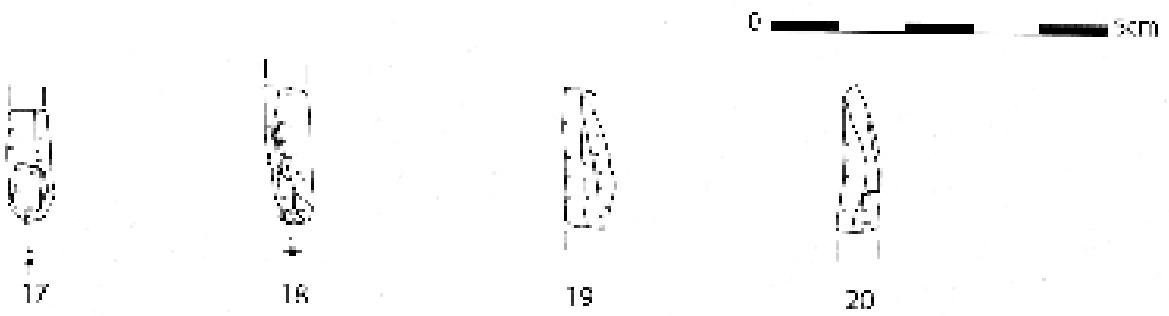

Figure 6: Lames et lamelles brutes. Figure 6: Primary blades and bladelets. ment secondaire en fin de débitage pour pallier le manque de convexités. C'est tout de même ce problème qui signe l'abandon des nucléus;

- débitage mené, sur des galets marins, par une succession de changements d'axes de débitage, sur une même surface ou sur des tables sécantes; grâce à cette méthode, le débitage en lui-même assure, tout au moins en partie, les corrections permanentes qui sont nécessaires;

- gestion du volume identique mais qui présente un stade d'exhaustion poussé; ainsi, il n'existe plus de surface corti- cale et les dimensions finales des nucléus (jusqu'à 20 x 20 x $10 \mathrm{~mm}$ ) poussent à s'interroger sur leur mode de préhension, les techniques de débitage ainsi que la destination des supports.

\section{De l'Azilien au Néolithique : comment rendre à César ce qui appartient à César?}

Létude technologique a offert des éléments de diagnose complémentaires à l'étude typologique. Ainsi, hormis la 


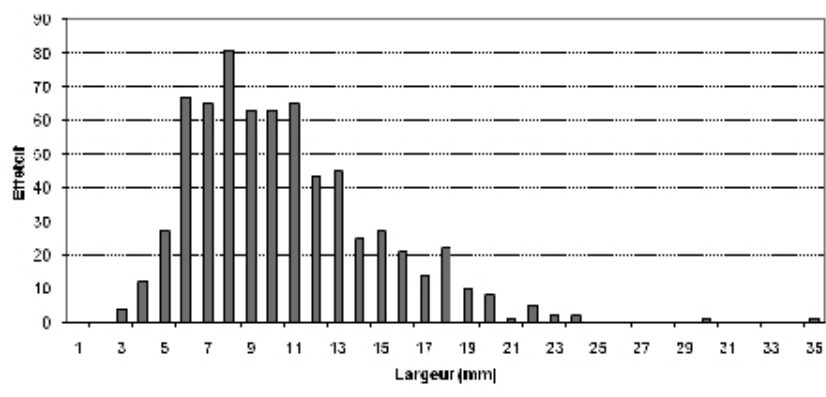

Figure 7 : Objectifs du débitage : largeurs.

Figure 7: Debitage aims: widths.

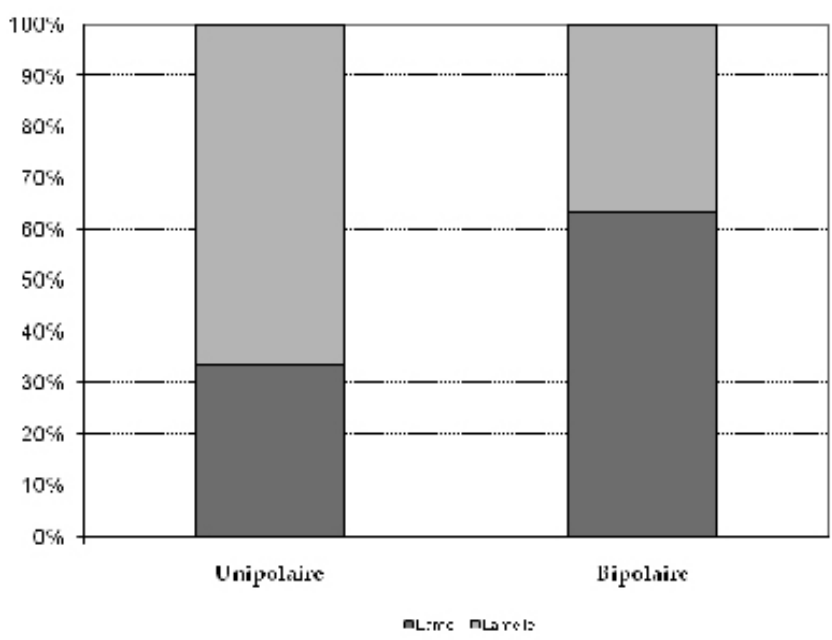

Figure 8 : Taux de bipolarité selon le support.

Figure 8: Bipolarity rates according to blanks.

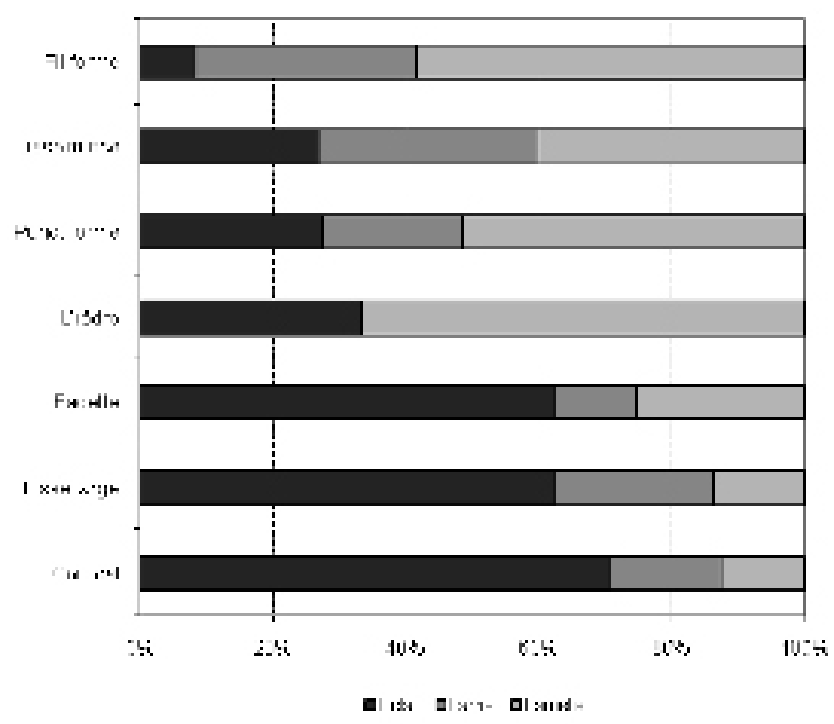

Figure 9 : Répartition des talons en fonction des supports. Figure 9: Platform frequency according to blanks.

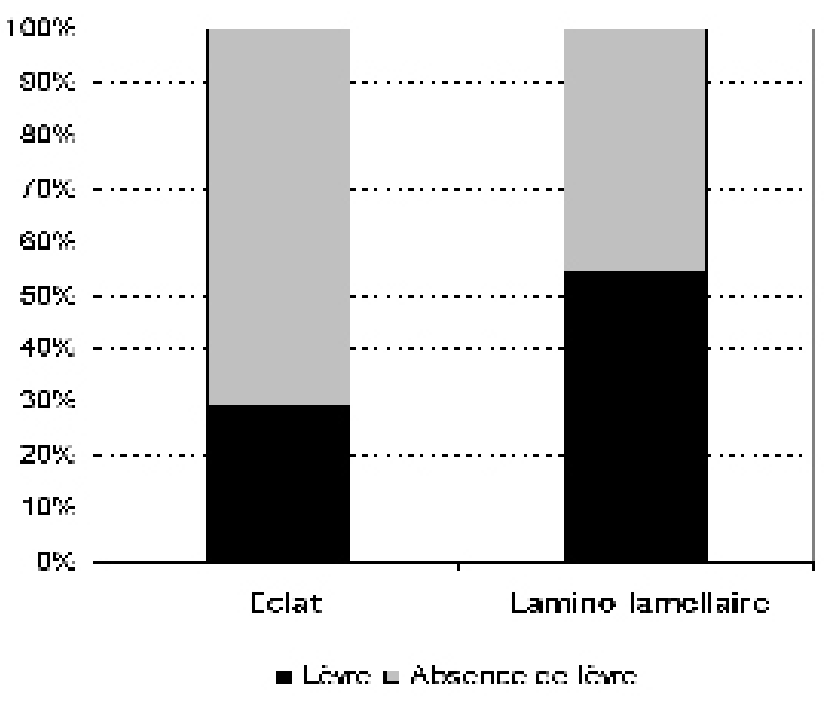

Figure 10 : Proportion de lèvres selon les supports.

Figure 10: Core reduction according to blanks.

rareté des outils caractéristiques du Néolithique, la quasiabsence de produits portant les stigmates de percussion sur enclume confirme la faible part de ces industries en dehors des cairns (Guyodo et Marchand, 2005; Donnart et al., 2009). La phase d'occupation initiale du site, perçue à travers la présence de quelques monopointes à dos courbe aziliennes s'exprime également dans le débitage comme en témoignent les lames et éclats laminaires souvent bipolaires et peu réguliers. Le faible degré de préparation au détachement des produits, qui s'illustre dans les exploitations de la phase récente de l'Azilien régional (Marchand et al., 2004), mais aussi dans le reste de l'aire d'influence azilienne (Valentin, 1995), est également observable sur ces pièces. Cette composante rappelle d'ailleurs l'industrie du site de Lann-Gazel (Trémaouézan, Finistère : Le Goffic, 2001).

Le premier Mésolithique est bien connu dans le Finistère par le groupe de Bertheaume (Gouletquer, 1973; Gouletquer et al., 1996; Kayser, 1991 ; Marchand, 2005; Blanchet et al., 2006). Rattaché plus précisément au Mésolithique moyen, ce techno-complexe est caractérisé par des armatures hypermicrolithiques telles que les lamelles de type Bertheaume, les triangles scalènes étroits et les pointes à bord abattu avec ou sans base retouchée. À Guennoc, les pointes de projectiles ne rappellent pas ce techno-complexe. De plus, le débitage sur éclat, fréquent dans le Mésolithique moyen finistérien, n'a pas été pratiqué sur l'îlot. Les nucléus ne mesurant que quelques millimètres en fin d'exploitation évoquent par contre ceux de la Petite-Île (Pontchâteau, Loire-Atlantique : Marchand et al., 1998) même si, dans le cas présent, le changement d'axe de débitage n'implique pas souvent une exploitation bipolaire 

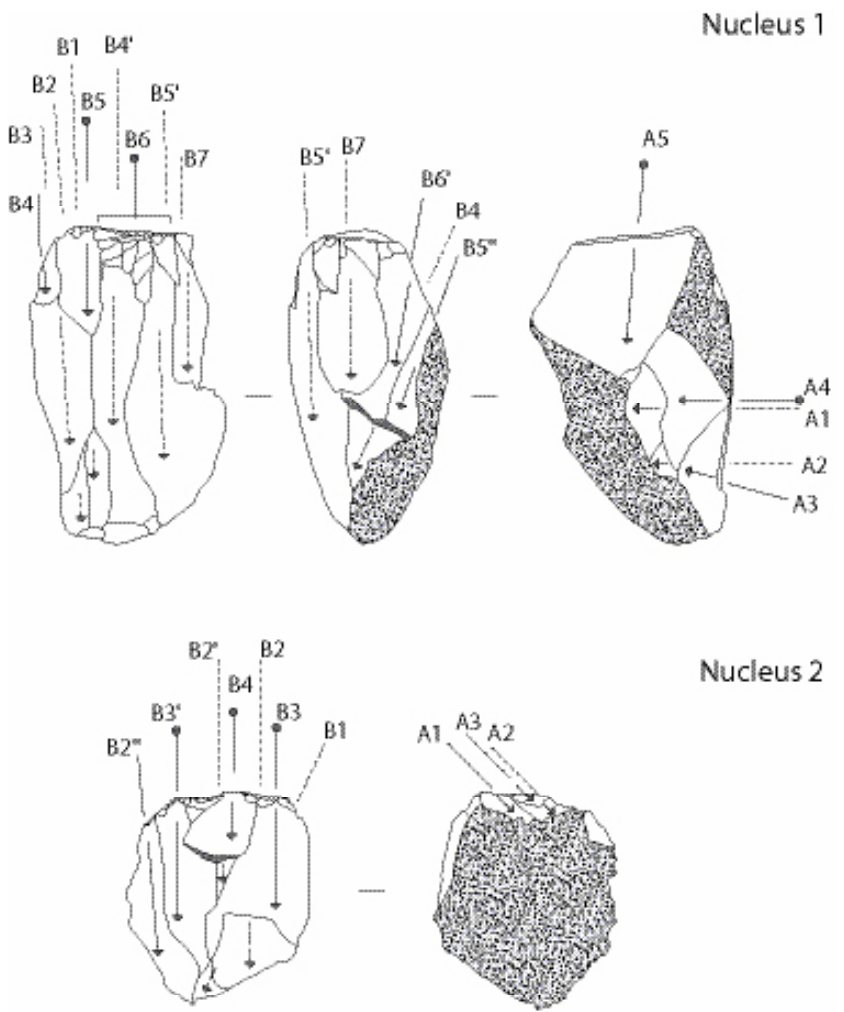

Nucleus 2
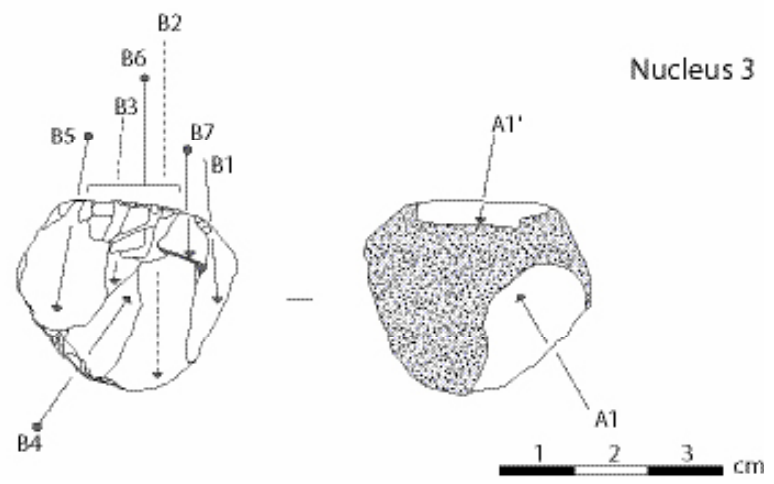

Figure 11 : Schémas diacritiques de nucleus. 1 : nucleus à lamelles, avec une table gérée depuis un plan de frappe et un dos cortical; 2 et 3 : nucleus à lamelles et éclats lamellaires, avec une table gérée depuis un plan de frappe (éventuelle intervention d'une surface de percussion secondaire) et un dos cortical. Les lettres correspondent aux séquences, les chiffres à l'ordre des enlèvements au sein de chacune d'entre elles.

Figure 11: Diacritic schemae of core reduction.

séquentielle. Tout cela nous invite à placer cette occupation au tout début du Mésolithique.

Les autres sites relevant du Mésolithique ancien en Finistère d'après l'inventaire inédit de P. Gouletquer et al. Toulanay-1 (Plogonnec), Parc-Balan (Plouigneau) et Cougnar-Zac'h (Santec) - présentent une industrie qui rappelle celle de Guennoc. Peut-être faudrait-il parler d'une phase de transition entre industrie post-azilienne et Mésolithique comme le suggèrent les lamelles à dos rectiligne apparentées aux pointes des Blanchères et les pointes à troncature oblique, ainsi que l'avait proposé O. Kayser (1984). L'avancée des recherches et les risques importants de mélanges nous oblige à ne conserver cette idée que comme pure hypothèse.

\section{INSTALLATION, ENVIRONNEMENT, SUBSISTANCE ET CONSÉQUENCES SUR L'ORGANISATION SOCIO-ÉCONOMIQUE}

Durant les phases anciennes d'occupation de Guennoc, le domaine maritime stricto sensu était situé à quelques kilomètres tout comme la zone estuarienne de l'Aber Wrac'h, celle de l'Aber Benoît étant en revanche à proximité directe du site (fig. 1). Par conséquent, ces chasseurs-collecteurs ont eu l'opportunité d'exploiter deux biotopes différents, avec un fort potentiel en ressources alimentaires. En plus de cette abondance, de tels milieux bénéficient d'une forte stabilité, contrairement aux biotopes terrestres des mêmes latitudes, et sont moins sujets aux phénomènes de saisonnalité (Dunbar, 1960). La forte production primaire des estuaires (micro-organismes, tant animaux que végétaux, à la base de la chaîne alimentaire) (Odum, 1971; Lieth et Whittaker, 1975), attire de nombreuses espèces de poissons qui y trouvent quantité de nutriments. Il s'agit également d'un lieu de passage pour les poissons migrateurs tels que le saumon, l'alose ou l'anguille. L'association d'un milieu estuarien et d'un littoral rocheux permet aussi le développement d'une multitude d'espèces de mollusques et de crustacés et la transgression flandrienne a certainement favorisé la création de bancs de coquillages sur les plaines inondées (Yesner et al., 1980). Les ressources marines constituent une excellente source de calcium, d'iode, et de sels minéraux, mais sont en revanche assez pauvre en calories, à l'exception des poissons gras et des mammiferes marins (ibid.). L'investissement en matière de recherche, d'acquisition et de traitement de ces ressources est cependant plus faible que pour les mammifères terrestres, ce qui augmente leur taux de retour (Kcal acquises par heure). Ces activités de collecte au sens large peuvent être complétées par la chasse des oiseaux; en effet, les estuaires sont aussi connus pour être des refuges prisés d'espèces migratrices ou hivernantes qui s'ajoutent à la longue liste des oiseaux marins des littoraux de la région. Cette richesse du milieu maritime n'interdit pas non plus le recours aux ressources terrestres tant animales que végétales.

Nous ne pouvons finir ce tour d'horizon des ressources disponibles sans évoquer la matière première lithique. Absent en position primaire sur le Massif armoricain, le silex peut 


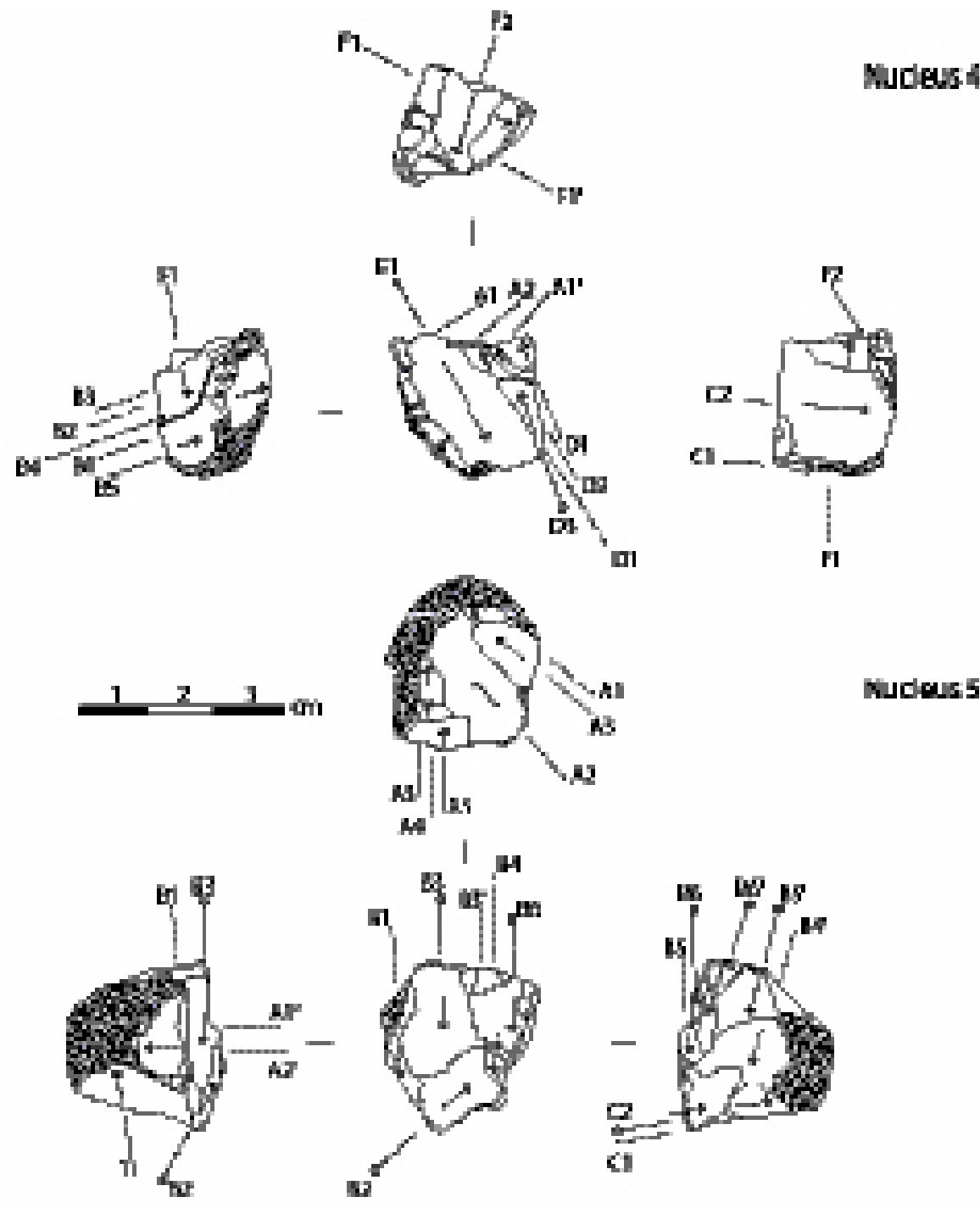

Figure 12 : Schémas diacritiques de nucléus (suite). 4 \& 5 : nucleus à lamelles et éclats lamellaires, exploitation sur plusieurs tables avec changements d'axe de débitage; 6 : nucleus à éclats. Les lettres correspondent aux séquences, les chiffres à l'ordre des enlèvements au sein de chacune d'entre elles.

Figure 12: Diacritic schemae of core reduction (continued).

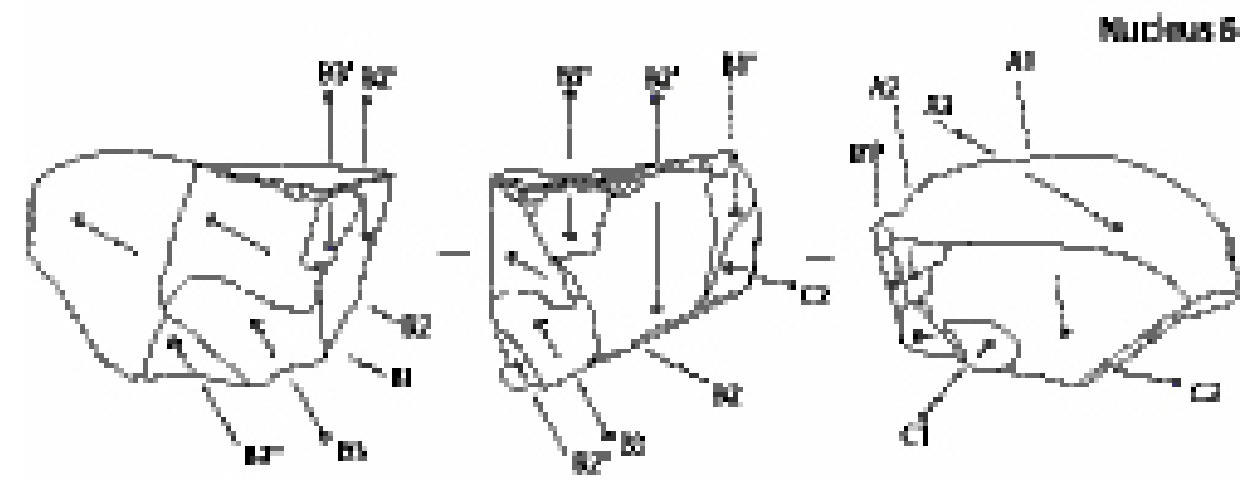

être collecté sous la forme de galets dans des cordons côtiers. Ainsi les chasseurs-collecteurs de Guennoc ont pu s'approvisionner en matériaux siliceux à proximité immédiate de leur habitat, ce qui n'exclut pas des apports plus lointains. Ces environnements étaient facilement accessibles lors des activités de subsistance journalières étant donné leur faible distance. Les études ethnographiques montrent que les groupes de chasseurs-collecteurs exploitent leur environnement dans un rayon d'environ $6 \mathrm{~km}$; passé cette distance, ils préfèrent plutôt déplacer le campement (Endicott et Endicott, 1986, p. 149; Harako, 1981, p. 535; Rai, 1990 ; Vincent, 1984; Williams, 1974, p. 74). En plus de la position de 

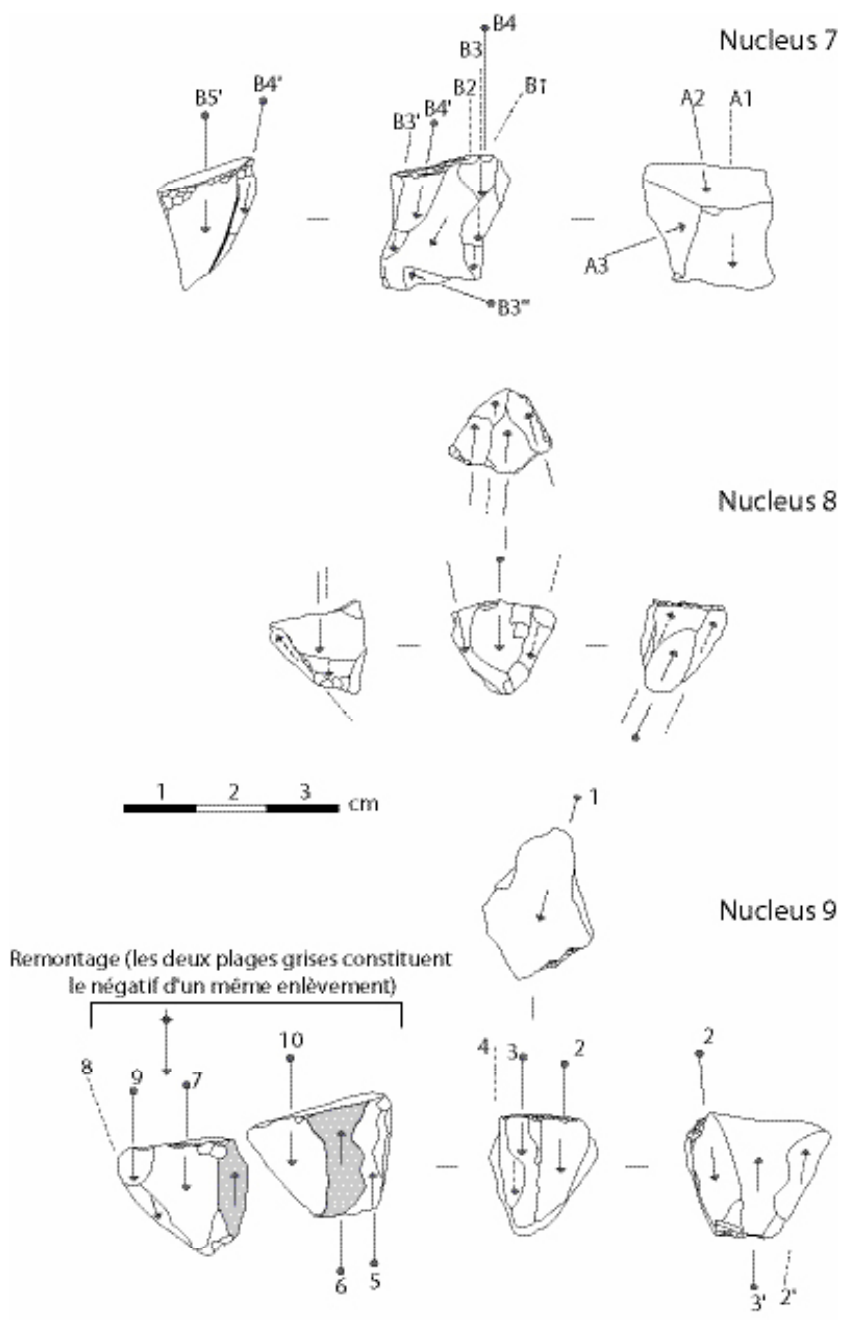

Figure 13 : Schémas diacritiques de nucleus (suite). 7, 8, 9 : nucleus à lamelles et éclats lamellaires; exploitation sur plusieurs tables avec changements d'axe de débitage, stade d'exhaustion poussé. Les lettres correspondent aux séquences, les chiffres à l'ordre des enlèvements au sein de chacune d'entre elles.

Figure 13: Diacritic schemae of core reduction (continued).

promontoire, l'abondance en ressources animales, végétales et minérales a donc certainement joué un rôle dans la pérennité de l'occupation de l'Île Guennoc.

Dans le cadre d'une mobilité à caractère essentiellement résidentiel (Binford, 1980), une telle diversité et disponibilité annuelle des ressources dans ces environnements permettrait aux groupes de chasseurs-collecteurs de pallier le manque de gibier en domaine terrestre durant les mois d'hiver : "The major effect of this diversity on the coastal population is that, during the most critical parts of the year, when the biomass of preferred resources is low, alternate forms of sustenance exist as a buffer" (Yesner et al., 1980, p. 729). Cependant, les exemples ethnographiques montrent que les installations en milieu côtier impliquent géné- ralement une mobilité de type plutôt logistique (Binford, 1980). D'après les données de L. Binford (2001), nous avons calculé qu'environ $71 \%$ des groupes de chasseurs-collecteurs actuels et sub-actuels de la planète dont la pêche représente plus de $50 \%$ du régime alimentaire basaient leur économie sur une mobilité de type logistique. D'après les mêmes sources, les territoires des groupes en question sont plus réduits que ceux des sociétés essentiellement (à plus de $50 \%$ ) basées sur la chasse : $305,1 \mathrm{~km}^{2}$ contre $858,6 \mathrm{~km}^{2}$. À Guennoc, l'absence de restes fauniques conservés pour cette période ainsi que l'hétérogénéité de la série lithique restent cependant des obstacles à la compréhension des modalités des différentes phases d'occupation de l'île.

\section{Conclusions}

Depuis la fin des années 1980, la dynamique des recherches concernant les sociétés de la fin du Pléistocène et du début de l'Holocène, mise en parallèle avec le développement de nouvelles méthodes d'analyses comme la technologie lithique, a renouvelé nos connaissances des groupes de chasseurscollecteurs. L'analyse typologique de l'ensemble lithique de Guennoc avait déjà permis d'y repérer une composante azilienne; l'approche technologique affine le diagnostic en confirmant également l'existence d'une phase d'occupation ultérieure attribuable au premier Mésolithique. Depuis quelques années, après s'être développées en Amérique du Nord à l'initiative de L. Binford, les approches socio-économiques de ces sociétés se multiplient en Europe. Profitant de cet élan, nous nous sommes permis d'émettre plusieurs hypothèses quant aux conséquences de l'évolution des milieux sur les modes de vie des occupants de l'île Guennoc en nous appuyant sur des données ethnographiques.

\section{Bibliographie}

Allard, M., 1982 - Un habitat de chasseurs au camp d'Auvours. Actes du colloque International de Roanne-Villerest: Les habitats du Paléolithique supérieur, 22-24 juin 1982, p. 77-79 (inédit).

BINFORD, L.-R., 1980 - Willow Smoke and Dogs'tail: huntergatherer settlement systems archaeological site formation, American Antiquity, 45-1, p. 4-20.

Binford, L. R., 2001 - Constructing Frames of Reference: An Analytical Method for Archaeological Theory Building Using Ethnographic and Environmental Data Sets, Berkeley, Los Angeles/London, California Press, 563 p.

Blanchet, S., Kayser, O., Marchand, G. et Yven, E., 2006 - Le Mésolithique moyen en Finistère : de nouvelles datations pour le groupe de Bertheaume, Bulletin de la Société préhistorique française, 103, p. 507-518. 
Blanchet, F., Michel, S. et Naudinot, N., 2007 - Les Prises : Gisement azilien et mésolithique à Brizambourg (Charente-Maritime) (rapport d'évaluation), Poitiers, Direction régionale des Affaires Culturelles de Poitou-Charentes, Service régional de l'Archéologie, $77 \mathrm{p}$.

DAIre, M.-Y., 2008 - Des gaulois sur l'île de Guennoc, Revue archéologique de l'Ouest, 25, p. 93-137.

Donnart, K., Naudinot, N. et Le Clézio, L., 2009 - Approche expérimentale du débitage bipolaire sur enclume : caractérisation des produits et analyses des outils de production, Bulletin de la Société préhistorique française, 106, 3, p. 517-533.

Dunbar, M. J., 1960 - The Evolution of Stability in Marine Environments: Natural Selection at the Level of the Ecosystem, American Naturalist, 94, p. 129-136.

Endicott, K. et Endicotr, K. L., 1986 - The Question of Hunter-Gatherers Territoriality: The Case of the Batek in Malaysia, in Biesele, M., Gordon, R. and Lee, R. B. (eds.), The Past and Future of Kung Ethnography: Critical Reflexions and Symbolic Perspectives, Essays in Honour of Lorna Marshall, Hambourg, Helmut Buske Verlag, p. 137-162.

Giot, P.-R., HallÉgouËt, B. et MonNIER, J.-L., 1977 - Le Paléolithique supérieur du Pays de Léon (Finistère). Les Gisements de Roc'hToul, Parc-ar-Plenen (Guiclan), Enez Guennoc (Landéda) et la Forest-Landerneau, L'Anthropologie, 81, p. 201-234.

Giot, P.-R., 1987 - Barnenez, Carn, Guennoc, Rennes, Travaux du Laboratoire « Anthropologie - Préhistoire - Protohistoire - Quaternaire armoricain », 2 vol.

GouletQuer, P., 1973 - Découverte d'une nouvelle industrie mésolithique en Bretagne Occidentale, in KozlowsKi, S. K. (dir.), The Mesolithic in Europe. Varsovie, Warsaw University Press, p. 187-207.

Gouletquer, P., Kayser, O., Le Goffic, M., Léopold, P., Marchand, G. et Moullec, J.-M., 1996 - Où sont passés les Mésolithiques côtiers bretons? Bilan 1985-1995 des prospections de surface dans le Finistère, Revue archéologique de l'Ouest, 13, p. 5-30.

GouletQuer, P. et al., Inédit - Base de donnée « Mésolithique finistérien ", Rennes, Service régional de l'Archéologie de Bretagne.

Guyodo, J.-N. et MARChand, G., 2005 - La percussion bipolaire sur enclume dans l'ouest de la France de la fin du Paléolithique au Chalcolithique : une lecture économique et sociale, Bulletin de la Société préhistorique française, 102, p. 539-549.

Harako, R., 1981 - The Cultural Ecology of Hunting Behavior among Mbuti Pygmies in the Ituri Forest, Zaire, in Harding, R. and Teleki, G. (eds.), Omnivorous Primates, New York, Columbia University Press. p. 499-555.

Kayser, O., 1984 - Autour du Mésolithique en Bretagne, Revue archéologique de l'Ouest, 1, p. 7-13.

KaYSER, O., 1991 - Le Mésolithique breton : un état des connaissances en 1988. Mésolithique et néolithisation en France et dans les régions limitrophes (Actes du $113^{\mathrm{e}}$ Congrès national des Sociétés savantes), Paris, CTHS, p. 197-211.
LE Goffic, M., 2001 - Tremaouezan, Lann-Gazel, in Notices d'archéologie finistérienne pour 2001. Bulletin de la Société archéologique du Finistère, 130, p. 98-100.

Lieth, H. et WhitTaKeR, R.H., 1975 - Primary Productivity of the Biosphere. New York, Springer, 339 p.

Marchand, G., Gallais, J.-Y. et Mens, E., 1998 - Les industries à microlithes entre Loire et Vilaine. Revue archéologique de l'Ouest, 15, p. 15-28.

Marchand, G., Blancher, S., Chevallier, G., Gallais, J.-Y., Goffic, M. L., Naudinot, N. et Yven, E., 2004 - La fin du Tardiglaciaire sur le Massif armoricain : territoires et cultures matérielles. Paléo, 16, p. 137-170.

MarChand, G., 2005 - Les occupations mésolithiques à l'intérieur du Finistère. Bilan archéographique et méthodologique (20012003). Revue archéologique de l'Ouest, 22, p. 25-84.

Menier, D., 2004 - Morphologie et remplissage des vallées fossiles sud-armoricaines : apport de la stratigraphie sismique (thèse de doctorat). Lorient, Université de Bretagne Sud. 202 p.

Monnier, J.-L., 1980 - Le Paléolithique de la Bretagne dans son cadre géologique. Rennes, Université de Rennes 1, Travaux du laboratoire d'Anthropologie-Préhistoire-Protohistoire et Quaternaire armoricains, $608 \mathrm{p}$.

Naudinot, N., 2008 - Les armatures lithiques tardiglaciaires dans l'ouest de la France : Proposition d'organisation chronoculturelle et chaîne opératoire de fabrication, in Petillon, J.M., Dias-Meirinho, M.-H., Cattelain, P., Honegger M., Normand, C. et Valdeyron, N. (coord.), Recherches sur les armatures de projectiles du Paléolithique supérieur au Néolithique (actes du colloque C83, XIV congrès de l'UISPP, Lisbonne, 49 septembre 2006), Palethnologie, 1, p. 241-268.

Odum, E.-P., 1971 - Fundamentals of Ecology, Philadephia, Saunders, $383 \mathrm{p}$.

RaI, N., 1990 - Living in a Lean-To: Philippine Negrito Foragers in Transition. Chicago, University of Michigan, Museum of Anthropology, Anthropological Papers, Ann Arbor, 80, 184 p.

Rozoy, J.-G., 1978 - Les derniers chasseurs. L'épipaléolithique en France et en Belgique. Essai de synthèse, Hors série du Bulletin de la société archéologique champenoise, 3 vol.

VAlENTIN, B., 1995 - Les groupes humains et leurs traditions au Tardiglaciaire dans le Bassin parisien. Apport de la technologie lithique comparée (thèse de doctorat), Paris, Université de Paris $1,3 \mathrm{Vol}$.

Valentin, B. et Hantaï, A., 2005 - Transformations de l'industrie lithique pendant l'Azilien. étude des niveaux 3 et 4 du BoisRagot, in Chollet, A. et Dujardin, V., La Grotte du BoisRagot à Gouex (Vienne) - Magdalénien et Azilien - Essais sur les hommes et leur environnement, Paris, Société préhistorique française (mémoire 38), p. 89-182.

Vincent, A., 1984 - Plant Foods in Savanna Environments: A Preliminary Report of Tubers Eaten by the Hadza of Northern Tanzania, World Archaeology, 17, p. 131-147. 
Williams, B., 1974 - A Model Band Society, Washington, D.C., Society for American Archaeology, 29.

Yesner, D., Ayres, W., Carlson, D., Davis, R., Dewar, R., Gonzales Morales, M., Hassan, F.,Hayden, B., Lischka, J.,
Sheets, P., Osborn, A., Pokotylo, D., Rogers, T., SPanier, T., Turner, B. and Wreschner, E., 1980 - Maritime HunterGatherers: Ecology and Prehistory, Current Anthropology, 21, p. 727-750. 